\title{
Soft filter
}

\author{
Şaziye Yüksel · Naime Tozlu $\cdot$ Zehra Güzel Ergül
}

Received: 4 July 2013/ Accepted: 9 April 2014/Published online: 1 May 2014

(C) The Author(s) 2014. This article is published with open access at Springerlink.com

\begin{abstract}
We have studied some soft neighbourhood properties in a soft topological space and introduced soft filters which are defined over an initial universe with a fixed set of parameters. We have set up a soft topology with the help of a soft filter. We also have introduced the concepts of the greatest lower bound and the least upper bound of the family of soft filters over an initial universe, soft filter subbase and soft filter base. Also, we have explored some basic properties of these concepts.
\end{abstract}

Keywords Soft topological space $\cdot$ Soft neighbourhood system $\cdot$ Soft filter

\section{Introduction}

Mathematics is based on exact concepts and there is not vagueness for mathematical concepts. Since in many other fields such as medicine, engineering, economics and sociology, the notions are vague, researchers need to define some new concepts for vagueness. To deal with the these problems in real life, researchers proposed several methods

\footnotetext{
Ş. Yüksel (ه)

Department of Mathematics, Science Faculty, Selcuk University, Konya, Turkey

e-mail: syuksel@selcuk.edu.tr

N. Tozlu

Department of Mathematics, Science and Art Faculty, Nigde

University, Nigde, Turkey

e-mail: naimetozlu@nigde.edu.tr

Z. G. Ergül

Department of Mathematics, Science and Art Faculty, Ahi Evran

University, Kirsehir, Turkey

e-mail: zguzel@ahievran.edu.tr
}

such as fuzzy set theory, rough set theory and soft set theory. Fuzzy set theory [1] proposed by Zadeh in 1965 provides an appropriate framework for representing and processing vague concepts. The basic idea of fuzzy set theory hinges on fuzzy membership function. By fuzzy membership function, we can determine the belonging of an element to set to a degree. Rough set theory [2] which is proposed by Pawlak in 1982 is another mathematical approach to vagueness to catch the granularity induced by vagueness in information systems. It based on equivalence relation. The advantage of rough set method is that it does not need any additional information about data, like membership in fuzzy set theory.

Theory of fuzzy sets and theory of rough sets can be considered as tools for dealing with vagueness but both of these theories have their own difficulties. The reason for these difficulties is, possibly, the inadequacy of the parametrization tool of the theory as mentioned by Molodtsov [3] in 1999. Soft set theory [3] was initiated by Molodtsov as a completely new approach for modeling vagueness and uncertainty. According to Molodtsov [3, 4], the soft set theory has been successfully applied to many fields, such as functions smoothness, game theory, Riemann integration, theory of measurement and so on. $\mathrm{He}$ also showed how soft set theory is free from the parametrization inadequacy syndrome of fuzzy set theory, rough set theory, probability theory and game theory. Soft systems provide a very general framework with the involvement of parameters. Research works on soft sets are progressing rapidly in recent years.

Topological structure of soft sets was studied by many authors: Shabir and Naz [5] introduced the soft topological spaces which are defined over an initial universe with a fixed set of parameters. They studied the concepts of soft open set, soft interior point, soft neighbourhood of a 
point, soft separation axioms and subspace of a soft topological space. As a different approach to soft topology Çağman et al. [6] defined the concepts of soft open set, soft interior, soft closure, soft limit point, soft Hausdorff space. Aygünoğlu and Aygün [7] defined soft continuity of soft mapping, soft product topology and studied properties of soft projection mappings, soft compactness and generalized Tychonoff theorem to the soft topological space. Min [8] gave some results on soft topological spaces. Zorlutuna et al. [9] also investigated soft interior point, soft neighbourhood and soft continuity. They introduced the relationships between soft topology and fuzzy topology. Hussain and Ahmad [10] continued and discussed the properties of soft interior, soft exterior and soft boundary on soft topology. Varol and Aygün [11] defined convergence of sequences in soft topological space, diagonal soft set and studied the properties of soft Hausdorff space.

The main purpose of this paper is to introduce soft filters which are defined over an initial universe with a fixed set of parameters. First, we have given some basic ideas about soft sets, soft topological spaces and the results already studied. Then, we have discussed some basic properties of soft neighbourhoods. We have defined soft filters and studied some basic properties of soft filters. We have set up a soft topology with the help of a soft filter. We have introduced some new concepts in soft filters such as the greatest lower bound and the least upper bound of the family of soft filters, soft filter subbase and soft filter base. Also, we have explored some basic properties of these concepts.

\section{Preliminaries}

In this section, we present the basic definitions and results of soft set theory which may be found in earlier studies.

Let $X$ be an initial universe set and $E$ be the set of all possible parameters with respect to $X$. Parameters are often attributes, characteristics or properties of the objects in $X$. Let $P(X)$ denote the power set of $X$. Then, a soft set over $X$ is defined as follows.

Definition 1 [3] A pair $(F, A)$ is called a soft set over $X$ where $A \subseteq E$ and $F: A \rightarrow P(X)$ is a set valued mapping. In other words, a soft set over $X$ is a parameterized family of subsets of the universe $X$. For $\forall \varepsilon \in A, F(\varepsilon)$ may be considered as the set of $\varepsilon$-approximate elements of the soft set $(F, A)$. It is worth noting that $F(\varepsilon)$ may be arbitrary. Some of them may be empty, and some may have nonempty intersection.

Example 1 [12] Miss Zeynep and Mr. Ahmet are going to marry and they want to hire a wedding room. The soft set
$(F, E)$ describes the "capacity of the wedding room". Let $X=\left\{u_{1}, u_{2}, u_{3}, u_{4}, u_{5}, u_{6}\right\}$ be the wedding rooms under consideration, and $E=\left\{e_{1}=\right.$ big, $e_{2}=$ central, $e_{3}=$ cheap, $e_{4}=$ quality, $e_{5}=$ elegant $\}$ be the parameter set, $F\left(e_{1}\right)=\left\{u_{2}, u_{4}\right\}, F\left(e_{2}\right)=\left\{u_{1}, u_{3}, u_{4}\right\}, F\left(e_{3}\right)=\emptyset, F\left(e_{4}\right)$ $=\left\{u_{1}, u_{3}, u_{5}\right\}, F\left(e_{5}\right)=\left\{u_{1}, u_{6}\right\}$. The $\operatorname{soft} \operatorname{set}(F, E)$ is as follows: $(F, E)=\left\{e_{1}=\left\{u_{2}, u_{4}\right\}, e_{2}=\left\{u_{1}, u_{3}, u_{4}\right\}, e_{3}=\right.$ $\left.\emptyset, e_{4}=\left\{u_{1}, u_{3}, u_{5}\right\}, e_{5}=\left\{u_{1}, u_{6}\right\}\right\}$.

Definition 2 [13] A soft set $F_{A}$ on the universe $X$ is defined by the set of ordered pairs $F_{A}=\left\{\left(e, f_{A}(e)\right)\right.$ : $\left.e \in E, f_{A}(e) \in P(X)\right\}$, where $f_{A}: E \rightarrow P(X)$, such that $f_{A}(e) \neq \emptyset$ if $e \in A \subseteq E$ and $f_{A}(e)=\emptyset$ if $e \notin A$. Here, $f_{A}$ is called an approximate function of the soft set $F_{A}$. The value of $f_{A}(e)$ may be arbitrary.

Note that the set of all soft sets over $X$ will be denoted by $\mathcal{S}(X, E)$.

Definition 3 [14] Let $F_{A} \in \mathcal{S}(X, E)$. If $f_{A}(e)=\emptyset$ for all $e \in E$, then $F_{A}$ is called an empty soft set, denoted by $F_{\Phi}$ or $\Phi . f_{A}(e)=\emptyset$ means that there is no element in $X$ related to the parameter $e \in E$. Therefore, we do not display such elements in the soft sets, as it is meaningless to consider such parameters.

Definition 4 [14] Let $F_{A} \in \mathcal{S}(X, E)$. If $f_{A}(e)=X$ for all $e \in A$, then $F_{A}$ is called an $A$-universal soft set, denoted by $F_{\tilde{A}}=\tilde{A}$. If $A=E$, then the $A$-universal soft set is called a universal soft set, denoted by $F_{\tilde{E}}=\tilde{E}$.

Definition 5 [5] Let $Y$ be a nonempty subset of $X$, then $\tilde{Y}$ denotes the soft set $Y_{E}$ over $X$ for which $Y(e)=Y$, for all $e \in E$. In particular, $X_{E}$ will be denoted by $\tilde{X}$.

Definition 6 [14] Let $F_{A}, G_{B} \in \mathcal{S}(X, E)$. Then $F_{A}$ is a soft subset of $G_{B}$, denoted by $F_{A} \sqsubseteq G_{B}$ if $f_{A}(e) \subseteq g_{B}(e)$, for all $e \in E$.

Definition 7 [14] Let $F_{A}, G_{B} \in \mathcal{S}(X, E)$. Then $F_{A}$ and $G_{B}$ are soft equal, denoted by $F_{A}=G_{B}$, if and only if $f_{A}(e)=$ $g_{B}(e)$ for all $e \in E$.

Definition 8 [14] Let $F_{A}, G_{B} \in \mathcal{S}(X, E)$. Then, the soft union $F_{A} \sqcup G_{B}$, the soft intersection $F_{A} \sqcap G_{B}$, and the soft difference $F_{A} \backslash G_{B}$ of $F_{A}$ and $G_{B}$ are defined by the approximate functions $h_{A \cup B}(e)=f_{A}(e) \cup g_{B}(e), h_{A \cap B}(e)=$ $f_{A}(e) \cap g_{B}(e), h_{A \backslash B}(e)=f_{A}(e) \backslash g_{B}(e)$, respectively, and the soft complement $F_{A}^{c}$ of $F_{A}$ is defined by the approximate function $f_{A^{c}}(e)=f_{A}^{c}(e)$, where $f_{A}^{c}(e)$ is the complement of the set $f_{A}(e)$; that is, $f_{A}^{c}(e)=X \backslash f_{A}(e)$ for all $e \in E$.

Definition 9 [9] Let $I$ be an arbitrary index set and $\left\{\left(F_{A}\right)_{i}\right\}_{i \in I}$ be a subfamily of $\mathcal{S}(X, E)$. 
(a) The union of these soft sets is the soft set $G_{C}$, where $g_{C}(e)=\cup_{i \in I}\left(f_{A}\right)_{i}(e)$ for each $e \in E$. We write $\sqcup_{i \in I}\left(F_{A}\right)_{i}=G_{C}$.

(b) The intersection of these soft sets is the soft set $H_{D}$, where $h_{D}(e)=\bigcap_{i \in I}\left(f_{A}\right)_{i}(e)$ for all $e \in E$. We write $\prod_{i \in I}\left(F_{A}\right)_{i}=H_{D}$.

Definition 10 [9] The soft set $F_{A} \in \mathcal{S}(X, E)$ is called a soft point in $\tilde{X}$, denoted by $e_{F}$, if for the element $e \in A$, $f_{A}(e) \neq \emptyset$ and $f_{A}\left(e^{\prime}\right)=\emptyset$ for all $e^{\prime} \in A-\{e\}$. The soft point $e_{F}$ is said to be in the soft set $G_{B}$, denoted by $e_{F} \tilde{\in} G_{B}$, if for the element $e \in A$ and $f_{A}(e) \subseteq g_{B}(e)$.

Proposition 1 [9] Let $e_{F} \tilde{\in} \tilde{X}$ and $G_{B} \sqsubseteq \tilde{X}$. If $e_{F} \tilde{\in} G_{B}$ then $e_{F} \underset{\notin}{\notin} G_{B}^{c}$.

Definition 11 [5] Let $\tilde{\tau} \sqsubseteq \mathcal{S}(X, E)$. A soft topology on $X$, denoted by $\tilde{\tau}$, is a collection of soft subsets of $X$ having the following properties:

(1) $\Phi, \tilde{X} \in \tilde{\tau}$

(2) If $F_{A}, G_{B} \in \tilde{\tau}$, then $F_{A} \sqcap G_{B} \in \tilde{\tau}$

(3) If $\left(F_{A}\right)_{\lambda} \in \tilde{\tau}, \forall \lambda \in \Delta$, then $\sqcup_{\lambda \in \Delta}\left(F_{A}\right)_{\lambda} \in \tilde{\tau}$

The pair $(X, \tilde{\tau})$ is called a soft topological space. Every member of $\tilde{\tau}$ is called a soft open set. A soft set $F_{A}$ is called soft closed in $(X, \tilde{\tau})$ if $F_{A}^{c} \in \tilde{\tau}$.

Definition 12 [9] A soft set $G_{B}$ in a soft topological space $(X, \tilde{\tau})$ is called a soft neighbourhood of the soft point $e_{F} \tilde{\in} \tilde{X}$ if there exists a soft open set $H_{C}$ such that $e_{F} \tilde{\in} H_{C} \sqsubseteq G_{B}$.

Remark 1 A soft set $G_{B}$ in a soft topological space $(X, \tilde{\tau})$ is not only a soft neighbourhood of the soft point $e_{F}$, it is also a soft neighbourhood for all soft points of the soft set $H_{C}$ by Definition 12 .

The neighbourhood system of a soft point $e_{F}$, denoted by $\mathcal{N}_{\tilde{\tau}}\left(e_{F}\right)$, is the family of all its soft neighbourhoods.

Theorem 1 [9] The neighbourhood system $\mathcal{N}_{\tau}\left(e_{F}\right)$ at $e_{F}$ in a soft topological space $(X, \tilde{\tau})$ has the following properties:

$\left.V_{1}\right)$ If $G_{B} \in \mathcal{N}_{\tilde{\tau}}\left(e_{F}\right)$, then $e_{F} \tilde{\in} G_{B}$,

$\left.V_{2}\right)$ If $G_{B} \in \mathcal{N}_{\tilde{\tau}}\left(e_{F}\right)$ and $G_{B} \sqsubseteq H_{C}$, then $H_{C} \in \mathcal{N}_{\tilde{\tau}}\left(e_{F}\right)$,

$\left.V_{3}\right)$ If $G_{B}, H_{C} \in \mathcal{N}_{\tilde{\tau}}\left(e_{F}\right)$, then $G_{B} \sqcap H_{C} \in \mathcal{N}_{\tilde{\tau}}\left(e_{F}\right)$,

$\left.V_{4}\right)$ If $G_{B} \in \mathcal{N}_{\tilde{\tau}}\left(e_{F}\right)$, then there is a $M_{D} \in \mathcal{N}_{\tilde{\tau}}\left(e_{F}\right)$ such that $G_{B} \in \mathcal{N}_{\tilde{\tau}}\left(e_{H}^{\prime}\right)$ for each $e_{H}^{\prime} \tilde{\in} M_{D}$.
Definition 13 Let $(X, \tilde{\tau})$ be a soft topological space and let $\mathcal{G}_{\tilde{\tau}}\left(e_{F}\right)$ be a family of some soft neighbourhoods of soft point $e_{F}$. If, for each soft neighbourhood $G_{B}$ of $e_{F}$, there exists a $H_{C} \in \mathcal{G}_{\tilde{\tau}}\left(e_{F}\right)$ such that $e_{F} \tilde{\in} H_{C} \sqsubseteq G_{B}$, then we say that $\mathcal{G}_{\tilde{\tau}}\left(e_{F}\right)$ is a soft neighbourhood base at $e_{F}$.

Theorem 2 Let $(X, \tilde{\tau})$ be a soft topological space and $G_{B} \in \mathcal{S}(X, E) . G_{B}$ is soft open if and only if it is a soft neighbourhood of each of its soft points.

Proof $\Longrightarrow$ If $G_{B}$ is a soft open set, then for each soft point $e_{F} \tilde{\in} G_{B}, e_{F} \tilde{\in} G_{B} \sqsubseteq G_{B}$. Since $G_{B} \in \tilde{\tau}, G_{B}$ is a soft neighbourhood of each of its soft points by Definition 12 .

$\Longleftarrow$ For each soft point $e_{F} \tilde{\in} G_{B}$, there exists a soft set $\left(H_{C}\right)_{e_{F}} \in \tilde{\tau}$ such that $e_{F} \tilde{\in}\left(H_{C}\right)_{e_{F}} \sqsubseteq G_{B}$. For each $e_{F} \tilde{\in} G_{B}$, since $e_{F} \tilde{\in}\left(H_{C}\right)_{e_{F}} \quad$ we obtain $e_{F} \tilde{\in} \underset{e_{F} \tilde{\in} G_{B}}{\sqcup}\left(H_{C}\right)_{e_{F}} \quad$ and $G_{B} \sqsubseteq \underset{e_{F}}{\sqcup} \underset{\tilde{\epsilon} G_{B}}{\sqcup}\left(H_{C}\right)_{e_{F}}$. Conversely, for each $e_{F} \tilde{\in} G_{B}$, since $\left(H_{C}\right)_{e_{F}} \sqsubseteq G_{B}$, we obtain $\underset{e_{F}}{\sqcup} \tilde{\in} G_{B}\left(H_{C}\right)_{e_{F}} \sqsubseteq G_{B}$. Consequently, we get $G_{B}=\underset{e_{F} \in \tilde{\in} G_{B}}{\sqcup}\left(H_{C}\right)_{e_{F}}$. Hence, $G_{B}$ is a soft open set by Definition 11.

Theorem 3 If for each soft point $e_{F} \tilde{\in} \tilde{X}$ there corresponds a family $\mathcal{N}\left(e_{F}\right)$ such that the properties $\left.\left.\left.V_{1}\right), V_{2}\right), V_{3}\right)$ and $\left.V_{4}\right)$ in Theorem 1 are satisfied, then there is a unique $\tilde{\tau}$ soft topological structure over $X$ such that for each $e_{F} \tilde{\in} \tilde{X}$, $\mathcal{N}\left(e_{F}\right)$ is the family of $\tilde{\tau}$-soft neighbourhoods of $e_{F}$.

Proof Let $\tilde{\tau} \quad=\left\{G_{B} \in \mathcal{S}(X, E): e_{F} \tilde{\in} G_{B} \Longrightarrow G_{B} \in\right.$ $\left.\mathcal{N}\left(e_{F}\right)\right\}$. It is clear that, $\tilde{\tau}$ is a soft topology over $X$. The family $\tilde{\tau}$ certainly satisfies axioms (3) and (2) in Definition 11: for (3), this follows immediately from $V_{2}$ ) in Theorem 1, and for (2), from $V_{3}$ ) in Theorem 1. The axiom (1) in Definition 11 is a result of $V_{2}$ ) and $V_{3}$ ) in Theorem 1. It remains to show that, in the soft topology defined by $\tilde{\tau}, \mathcal{N}\left(e_{F}\right)$ is the set of $\tilde{\tau}$-soft neigbourhoods of $e_{F}$ for each $e_{F} \tilde{\in} \tilde{X}$. It follows from $\left.V_{2}\right)$ in Theorem 1 that every $\tilde{\tau}$-soft neighbourhood of $e_{F}$ belongs to $\mathcal{N}\left(e_{F}\right)$. Conversely, let $\left(G_{B}\right)_{1}$ be a soft set belonging to $\mathcal{N}\left(e_{F}\right)$, and let $\left(G_{B}\right)_{2}$ be the soft set of soft points $e_{M} \tilde{\in} \tilde{X}$ such that $\left(G_{B}\right)_{1} \in \mathcal{N}\left(e_{M}\right)$. If we can show that $e_{F} \tilde{\in}\left(G_{B}\right)_{2}, \quad\left(G_{B}\right)_{2} \sqsubseteq\left(G_{B}\right)_{1}$ and $\left(G_{B}\right)_{2} \in \tilde{\tau}$, then the proof will be complete. Since for every soft point $e_{M} \tilde{\in}\left(G_{B}\right)_{2}$ belongs to $\left(G_{B}\right)_{1}$ by reason of $\left.V_{1}\right)$ in Theorem 1 and the hypothesis $\left(G_{B}\right)_{1} \in \mathcal{N}\left(e_{M}\right)$, we obtain $\left(G_{B}\right)_{2} \sqsubseteq\left(G_{B}\right)_{1}$. Since $\left(G_{B}\right)_{1} \in \mathcal{N}\left(e_{F}\right)$ and $\left(G_{B}\right)_{2}$ $\sqsubseteq\left(G_{B}\right)_{1}$, we have $e_{F} \tilde{\in}\left(G_{B}\right)_{2}$. It remains to show that 
$\left(G_{B}\right)_{2} \in \tilde{\tau}$, i.e. that $\left(G_{B}\right)_{2} \in \mathcal{N}\left(e_{M}\right)$ for each $e_{M} \tilde{\in}\left(G_{B}\right)_{2}$. If $e_{M} \tilde{\in}\left(G_{B}\right)_{2}$ then by $\left.V_{4}\right)$ in Theorem 1 there is a soft set $\left(G_{B}\right)_{3}$ such that for each $e_{H} \tilde{\in}\left(G_{B}\right)_{3}$ we have $\left(G_{B}\right)_{1} \in \mathcal{N}\left(e_{H}\right)$. Since $\left(G_{B}\right)_{1} \in \mathcal{N}\left(e_{H}\right)$ means that $e_{H} \tilde{\in}\left(G_{B}\right)_{2}$, it follows that $\left(G_{B}\right)_{3} \sqsubseteq\left(G_{B}\right)_{2}$ and, therefore, by $\left.V_{2}\right)$ in Theorem 1 , that $\left(G_{B}\right)_{2} \in \mathcal{N}\left(e_{M}\right)$.

\section{Soft filter}

Definition 14 Let $\tilde{\mathcal{F}} \sqsubseteq \mathcal{S}(X, E)$, then $\tilde{\mathcal{F}}$ is a called a soft filter on $X$ if $\tilde{\mathcal{F}}$ satisfies the following properties:

$$
\begin{aligned}
& \left.f_{1}\right) \Phi \notin \tilde{\mathcal{F}} \\
& \left.f_{2}\right) \forall F_{A}, G_{B} \in \tilde{\mathcal{F}} \Longrightarrow F_{A} \sqcap G_{B} \in \tilde{\mathcal{F}} \\
& \left.f_{3}\right) \forall F_{A} \in \tilde{\mathcal{F}} \text { and } F_{A} \sqsubseteq G_{B} \Longrightarrow G_{B} \in \tilde{\mathcal{F}}
\end{aligned}
$$

Remark $2 \tilde{\mathcal{F}}$ satisfies the following property from $\left.f_{1}\right)$ and $\left.f_{2}\right)$ : "The all finite intersections of soft sets of $\tilde{\mathcal{F}}$ are not soft empty".

Remark 3 It is clear that $\tilde{X} \in \tilde{\mathcal{F}}$ by $\left.f_{3}\right)$.

Example 2 The family $\tilde{\mathcal{F}}=\{\tilde{X}\}$ is a soft filter over $X$.

Example 3 Let $\Phi \neq F_{A} \in \mathcal{S}(X, E)$. Then, the family $\tilde{\mathcal{F}}_{F_{A}}=\left\{G_{B}: F_{A} \sqsubseteq G_{B} \in \mathcal{S}(X, E)\right\}$ is a soft filter over $X$ and it is called atomic soft filter.

Example 4 Let $\mathbb{N}$ be the natural numbers and $E$ be a finite set. Consider the family $\tilde{\mathcal{F}}=\left\{F_{A}: \underset{e \in E}{\cup} \mathbb{N} \backslash f_{A}(e)\right.$ is finite $\}$, then $\widetilde{\mathcal{F}}$ is a soft filter and it is called soft Frechet filter.

Example 5 Let $X$ be an infinite set and $E$ be a finite set. Consider the family $\widetilde{\mathcal{F}}=\left\{F_{A}: \cup_{e \in E} X \backslash f_{A}(e)\right.$ is finite $\}$, then $\tilde{\mathcal{F}}$ is a soft filter and it is called soft cofinite filter.

Example 6 Let $X$ be an uncountable set and $E$ be a countable set. Consider the family $\tilde{\mathcal{F}}=\left\{F_{A}\right.$ : $\cup_{e \in E} X \backslash f_{A}(e)$ is countable $\}$, then $\tilde{\mathcal{F}}$ is a soft filter and it is called soft cocountable filter.

Proposition 2 Let $\tilde{\mathcal{F}}$ be a soft filter over $X$. Then the collection

$\tilde{\mathcal{F}}_{e}=\left\{f_{A}(e): F_{A} \in \tilde{\mathcal{F}}\right\}$

for each $e \in E$, does not define a filter over $X$.
Proof If $f_{A}(e)=\emptyset$ for any $e \in E$ then $\emptyset \in \widetilde{\mathcal{F}}_{e}$. Hence $\widetilde{\mathcal{F}}_{e}$ is not a filter over $X$ in general topology.

Example 7 Let $X=\left\{x_{1}, x_{2}, x_{3}\right\}, E=\left\{e_{1}, e_{2}, e_{3}\right\}$ and $\tilde{\mathcal{F}}=\left\{\tilde{X},\left(F_{A}\right)_{1},\left(F_{A}\right)_{2},\left(F_{A}\right)_{3},\left(F_{A}\right)_{4}\right\}$ be a soft filter defined over $X$ where $\left(F_{A}\right)_{1}=\left\{\left(e_{1}, \emptyset\right),\left(e_{2}, \emptyset\right),\left(e_{3},\left\{x_{1}, x_{3}\right\}\right)\right\}$, $\left(F_{A}\right)_{2}=\left\{\left(e_{1}, \emptyset\right),\left(e_{2},\left\{x_{2}, x_{3}\right\}\right),\left(e_{3},\left\{x_{1}, x_{3}\right\}\right)\right\},\left(F_{A}\right)_{3}=\left\{\left(e_{1}\right.\right.$, $\left.\left.\left\{x_{1}, x_{2}\right\}\right),\left(e_{2}, \emptyset\right),\left(e_{3},\left\{x_{1}, x_{3}\right\}\right)\right\},\left(F_{A}\right)_{4}=\left\{\left(e_{1},\left\{x_{1}, x_{2}\right\}\right),\left(e_{2}\right.\right.$, $\left.\left.\left\{x_{2}, x_{3}\right\}\right),\left(e_{3},\left\{x_{1}, x_{3}\right\}\right)\right\}$. Since $\emptyset \in \tilde{\mathcal{F}}_{e_{1}}$, then $\tilde{\mathcal{F}}_{e_{1}}$ is not a filter over $X$.

Theorem 4 Let $(X, \tilde{\tau})$ be a soft topological space. The neighbourhood system $\mathcal{N}_{\tilde{\tau}}\left(e_{F}\right)$ is a soft filter for every $e_{F} \stackrel{\sim}{\in} \tilde{X}$. Also, it is called soft neighbourhoods filter of the soft point $e_{F}$.

Proof

$\left.f_{1}\right)$ By $\left.V_{1}\right)$ in Theorem 1, since $e_{F} \tilde{\in} G_{B}$, we obtain $\Phi \notin \mathcal{N}_{\tilde{\tau}}\left(e_{F}\right)$.

$f_{2}$ ) This is clearly seen by $V_{3}$ ) in Theorem 1 .

$f_{3}$ ) This is clearly seen by $V_{2}$ ) in Theorem 1 .

Now, we set up a soft topology with the help of a soft filter.

Theorem 5 If, for every $e_{F} \tilde{\in} \tilde{X}$, there exists a soft filter $\tilde{\mathcal{F}}\left(e_{F}\right)=\mathcal{N}_{\tilde{\tau}}\left(e_{F}\right)$ which satisfies the following two properties, then there exists an unique soft topology $\tilde{\tau}$ such that $\tilde{\mathcal{F}}\left(e_{F}\right)$ consists of the $\tilde{\tau}$-soft neighbourhoods of the soft point $e_{F}$.

$\left.V_{f_{1}}\right)$ Every soft set in the soft filter $\tilde{\mathcal{F}}\left(e_{F}\right)$ contains the soft point $e_{F}$,

$\left.V_{f_{2}}\right)$ For every $G_{B} \in \tilde{\mathcal{F}}\left(e_{F}\right)$ there exists a $H_{C} \in \tilde{\mathcal{F}}\left(e_{F}\right)$ such that for every $e_{M} \tilde{\in} H_{C}, G_{B} \in \tilde{\mathcal{F}}\left(e_{M}\right)$.

Proof Since the axioms $\left.\left.\left.\left.f_{1}\right), f_{2}\right), f_{3}\right), V_{f_{1}}\right)$ and $V_{f_{2}}$ ) are equivalent to the neighbourhood axioms $\left.V_{1}\right)-V_{4}$ ), by Theorem 3 , there exists a soft topology $\tilde{\tau}$ such that $\tilde{\mathcal{F}}\left(e_{F}\right)$ consists of the $\tilde{\tau}$-soft neighbourhoods of the soft point $e_{F}$.

Example 8 Let $(X, \tilde{\tau})$ be a soft topological space and $e_{F} \tilde{\in} \tilde{X}$. Since $G_{B}$ cannot be an element of $\mathcal{G}_{\tilde{\tau}}\left(e_{F}\right)$ for every $H_{C} \in \mathcal{G}_{\tilde{\tau}}\left(e_{F}\right)$ and $H_{C} \sqsubseteq G_{B}$, then the soft neighbourhood base $\mathcal{G}_{\tau}\left(e_{F}\right)$ is not a soft filter over $X$. 
Definition 15 Let $\tilde{\mathcal{F}}_{1}$ and $\tilde{\mathcal{F}}_{2}$ be soft filters over $X$. If $\tilde{\mathcal{F}}_{1} \sqsubseteq \tilde{\mathcal{F}}_{2}$, then $\tilde{\mathcal{F}}_{2}$ is soft finer than $\tilde{\mathcal{F}}_{1}$. If $\tilde{\mathcal{F}}_{1} \sqsubset \tilde{\mathcal{F}}_{2}$, then $\tilde{\mathcal{F}}_{2}$ is soft strictly finer than $\tilde{\mathcal{F}}_{1}$. If either $\tilde{\mathcal{F}}_{1} \sqsubseteq \tilde{\mathcal{F}}_{2}$ or $\tilde{\mathcal{F}}_{2} \sqsubseteq \tilde{\mathcal{F}}_{1}$, then $\tilde{\mathcal{F}}_{1}$ is comparable with $\tilde{\mathcal{F}}_{2}$.

Proposition 3 Let $\left(\tilde{\mathcal{F}}_{i}\right)_{i \in I}$ be a family of soft filters. Then, the family $\left(\tilde{\mathcal{F}}_{i}\right)_{i \in I}$ is partially ordered according to " $\sqsubseteq$ ".

Theorem 6 Let $\left(\tilde{\mathcal{F}}_{i}\right)_{i \in I}$ be a family of soft filters over $X$. Then $\tilde{\mathcal{F}}=\prod_{i \in I} \tilde{\mathcal{F}}_{i}$ is a soft filter over $X$.

Proof

$\left.f_{1}\right)$ Since $\Phi \notin \tilde{\mathcal{F}}_{i}$ for each $i \in I$, then $\Phi$ does not belong to $\tilde{\mathcal{F}}=\prod_{i \in I} \tilde{\mathcal{F}}_{i}$.

$f_{2)}$ Let $F_{A}, G_{B} \in \widetilde{\mathcal{F}}=\prod_{i \in I} \tilde{\mathcal{F}}_{i}$. Then, $F_{A}, G_{B} \in \widetilde{\mathcal{F}}_{i}$ for each $i \in I$. Since $F_{A} \sqcap G_{B} \in \tilde{\mathcal{F}}_{i}$ for each $i \in I$, so we obtain $F_{A} \sqcap G_{B} \in \tilde{\mathcal{F}}=\prod_{i \in I} \tilde{\mathcal{F}}_{i}$.

$\left.f_{3}\right)$ Let $F_{A} \in \widetilde{\mathcal{F}}=\prod_{i \in I} \tilde{\mathcal{F}}_{i}$ and $F_{A} \sqsubseteq G_{B}$. Since $F_{A} \in \tilde{\mathcal{F}}_{i}$ for each $i \in I$ and $F_{A} \sqsubseteq G_{B}$, we get $G_{B} \in \tilde{\mathcal{F}}_{i}$ for each $i \in I$. Hence $G_{B} \in \widetilde{\mathcal{F}}=\prod_{i \in I} \widetilde{\mathcal{F}}_{i}$.

Remark 4 The soft filter $\tilde{\mathcal{F}}$ in Theorem 6 is called the greatest lower bound of the family $\left(\tilde{\mathcal{F}}_{i}\right)_{i \in I}$.

Remark 5 The union of soft filters over $X$ is generally not a soft filter over $X$.

Example 9 Let $X=\left\{x_{1}, x_{2}, x_{3}\right\}, E=\left\{e_{1}, e_{2}\right\}$ and $\tilde{\mathcal{F}}_{1}=$ $\left\{\tilde{X},\left(F_{A}\right)_{1},\left(F_{A}\right)_{2},\left(F_{A}\right)_{3}\right\}, \quad \tilde{\mathcal{F}}_{2}=\left\{\tilde{X},\left(G_{B}\right)_{1},\left(G_{B}\right)_{2},\left(G_{B}\right)_{3}\right\}$ be two soft filters defined over $X$ where $\left(F_{A}\right)_{1}=\left\{\left(e_{1}\right.\right.$, $\left.\left.\left\{x_{1}\right\}\right),\left(e_{2},\left\{x_{2}, x_{3}\right\}\right)\right\},\left(F_{A}\right)_{2}=\left\{\left(e_{1},\left\{x_{1}, x_{2}\right\}\right),\left(e_{2}, X\right)\right\},\left(F_{A}\right)_{3}=$ $\left\{\left(e_{1},\left\{x_{1}\right\}\right),\left(e_{2},\left\{x_{2}\right\}\right)\right\}$ and $\left(G_{B}\right)_{1}=\left\{\left(e_{1},\left\{x_{3}\right\}\right),\left(e_{2},\left\{x_{1}, x_{2}\right\}\right)\right\}$, $\left(G_{B}\right)_{2}=\left\{\left(e_{1},\left\{x_{1}, x_{3}\right\}\right),\left(e_{2}, X\right)\right\},\left(G_{B}\right)_{3}=\left\{\left(e_{1},\left\{x_{1}, x_{3}\right\}\right),\left(e_{2}\right.\right.$, $\left.\left.\left\{x_{1}, x_{2}\right\}\right)\right\}$.Now, we define $\quad \tilde{\mathcal{F}}=\tilde{\mathcal{F}}_{1} \quad \cup \tilde{\mathcal{F}}_{2}=\{\tilde{X}$, $\left.\left(F_{A}\right)_{1},\left(F_{A}\right)_{2},\left(F_{A}\right)_{3},\left(G_{B}\right)_{1},\left(G_{B}\right)_{2},\left(G_{B}\right)_{3}\right\}$. If we take $\left(F_{A}\right)_{1}$ $\sqcap\left(G_{B}\right)_{1}=H_{C} \quad$ then $\quad h_{C}\left(e_{1}\right)=\left(f_{A}\right)_{1}\left(e_{1}\right) \cap\left(g_{B}\right)_{1}\left(e_{1}\right)=\left\{x_{1}\right\}$ $\cap\left\{x_{3}\right\}=\emptyset \quad$ and $\quad h_{C}\left(e_{2}\right)=\left(f_{A}\right)_{1}\left(e_{2}\right) \cap\left(g_{B}\right)_{1} \quad\left(e_{2}\right)=\left\{x_{2}, x_{3}\right\} \cap$ $\left\{x_{1}, x_{2}\right\}=\left\{x_{2}\right\}$ but $H_{C} \notin \tilde{\mathcal{F}}$. Hence $\tilde{\mathcal{F}}$ is not a soft filter over $X$.

Now, we investigate the least upper bound of the family of soft filters over $X$.
Proposition 4 Let $\widetilde{S} \sqsubseteq \mathcal{S}(X, E)$. Then there exists a soft filter $\tilde{\mathcal{F}}$ which contains the soft family $\tilde{S}$, if $\tilde{S}$ has the following property: "The all finite intersections of soft sets of $\tilde{S}$ are not soft empty”.

Proof Let

$\tilde{S}=\left\{\left(F_{A}\right)_{i}: \forall i \in J(J\right.$ is finite $\left.), \prod_{i \in J}\left(F_{A}\right)_{i} \neq \Phi\right\}$.

Then we give the family which consists of finite intersections of elements of $\tilde{S} ; \quad \tilde{\beta}=\left\{G_{B}: \forall i \in J\right.$ (J is finite), $\left(F_{A}\right)_{i} \in \widetilde{S}$ and $\left.G_{B}=\prod_{i \in J}\left(F_{A}\right)_{i}\right\}$.

Then, the family $\tilde{\mathcal{F}}(\tilde{S})=\left\{H_{C}: G_{B} \in \tilde{\beta}\right.$ and $\left.G_{B} \sqsubseteq H_{C}\right\}$ is a soft filter over $X$.

$\left.f_{1}\right)$ Since $\Phi \notin \tilde{\beta}$, for every $H_{C} \in \tilde{\mathcal{F}}(\tilde{S}), H_{C} \neq \Phi$ and so $\Phi \notin \tilde{\mathcal{F}}(\tilde{S})$.

$\left.f_{2}\right)$ Let $\left(H_{C}\right)_{1},\left(H_{C}\right)_{2} \in \tilde{\mathcal{F}}(\tilde{S})$. There exist soft sets $\left(G_{B}\right)_{1},\left(G_{B}\right)_{2} \in \tilde{\beta} \quad$ such that $\left(G_{B}\right)_{1} \sqsubseteq\left(H_{C}\right)_{1}$ and $\left(G_{B}\right)_{2} \sqsubseteq\left(H_{C}\right)_{2}$. From the definition of $\tilde{\beta}$, $\Phi \neq\left(G_{B}\right)_{1} \sqcap\left(G_{B}\right)_{2} \in \tilde{\beta}$. Since $\left(G_{B}\right)_{1} \sqcap\left(G_{B}\right)_{2} \sqsubseteq\left(H_{C}\right)_{1}$ $\sqcap\left(H_{C}\right)_{2}$, we obtain $\left(H_{C}\right)_{1} \sqcap\left(H_{C}\right)_{2} \in \tilde{\mathcal{F}}(\tilde{S})$.

$\left.f_{3}\right)$ Let $\left(H_{C}\right)_{1} \in \tilde{\mathcal{F}}(\tilde{S})$ and $\left(H_{C}\right)_{1} \sqsubseteq\left(H_{C}\right)_{2}$. Then, there exists a soft set $G_{B} \in \tilde{\beta}$ such that $G_{B} \sqsubseteq\left(H_{C}\right)_{1}$. Since $\left(H_{C}\right)_{1} \sqsubseteq\left(H_{C}\right)_{2}$, we obtain $\left(H_{C}\right)_{2} \in \tilde{\mathcal{F}}(\tilde{S})$.

Remark 6 The soft filter $\tilde{\mathcal{F}}(\tilde{S})$ in Proposition 4 is said to be generated by $\widetilde{S}$ and $\widetilde{S}$ is said to be soft filter subbase of $\tilde{\mathcal{F}}(\tilde{S})$. It is clear that, $\tilde{S} \sqsubseteq \tilde{\mathcal{F}}(\tilde{S})$.

Proposition 5 The soft filter $\tilde{\mathcal{F}}(\tilde{S})$ which is generated by $\tilde{S}$ is the coarsest soft filter which contains $\tilde{S}$.

Proof Suppose that $\tilde{S} \sqsubseteq \tilde{\mathcal{F}}_{1}$. By Proposition 4, $\tilde{S} \sqsubseteq \tilde{\beta} \sqsubseteq \tilde{\mathcal{F}}_{1}$. By Remark 6 , for every $H_{C} \in \tilde{\mathcal{F}}(\tilde{S})$, there exists a $G_{B} \in \widetilde{\beta}$ such that $G_{B} \sqsubseteq H_{C}$. Since $\tilde{\beta} \sqsubseteq \tilde{\mathcal{F}}_{1}$ then $G_{B} \in \tilde{\mathcal{F}}_{1}$. Since $\tilde{\mathcal{F}}_{1}$ is a soft filter, $H_{C} \in \tilde{\mathcal{F}}_{1}$ by $\left.f_{3}\right)$ in Definition 14. Hence we obtain $\tilde{\mathcal{F}}(\tilde{S}) \sqsubseteq \tilde{\mathcal{F}}_{1}$.

Theorem 7 The family $\left(\tilde{\mathcal{F}}_{i}\right)_{i \in I}$ of soft filters over $X$ has a least upper bound if and only if for all finite subfamilies $\left(\tilde{\mathcal{F}}_{i \leq i \leq n}\right.$ of $\left(\tilde{\mathcal{F}}_{i \in I}\right)_{i \in I}$ and all $\left(G_{B}\right)_{i} \in \tilde{\mathcal{F}}_{i}(1 \leq i \leq n)$, the intersection $\left(G_{B}\right)_{1} \sqcap \ldots \sqcap\left(G_{B}\right)_{n}$ is not soft empty. 
Proof $\Longrightarrow$ If there exists a least upper bound of the family $\left(\tilde{\mathcal{F}}_{i}\right)_{i \in I}$, by $\left.f_{1}\right)$ and $\left.f_{2}\right)$ in Definition 14 , for all finite subfamilies $\left(\tilde{\mathcal{F}}_{i}\right)_{1 \leq i \leq n}$ of $\left(\tilde{\mathcal{F}}_{i}\right)_{i \in I}$ and all $\left(G_{B}\right)_{i} \in \tilde{\mathcal{F}}_{i}$ $(1 \leq i \leq n)$, the intersection $\left(G_{B}\right)_{1} \sqcap \ldots \sqcap\left(G_{B}\right)_{n} \neq \Phi$.

$\Longleftarrow$ Let $\left(G_{B}\right)_{1} \sqcap \ldots \sqcap\left(G_{B}\right)_{n} \neq \Phi$ for all finite subfamilies $\left(\tilde{\mathcal{F}}_{i}\right)_{1 \leq i \leq n}$ of $\left(\tilde{\mathcal{F}}_{i}\right)_{i \in I}$ and all $\left(G_{B}\right)_{i} \in \tilde{\mathcal{F}}_{i}(1 \leq i \leq n)$. Then, the soft filter $\tilde{\mathcal{F}}(\tilde{S})$ generated by

$$
\widetilde{S}=\sqcup \tilde{\mathcal{F}}_{i \in I}=\left\{F_{A}:(\exists i \in I) F_{A} \in \widetilde{\mathcal{F}}_{i}\right\}
$$

is the least upper bound of the family $\left(\tilde{\mathcal{F}}_{i}\right)_{i \in I}$ by Proposition 5 .

Definition 16 Let $\widetilde{\beta} \sqsubseteq \mathcal{S}(X, E)$, then $\widetilde{\beta}$ is said to be a soft filter base on $X$ if

$$
\begin{aligned}
& \left.b_{1}\right) \tilde{\beta} \neq \Phi \text { and } \Phi \notin \widetilde{\beta}, \\
& \left.b_{2}\right) \forall F_{A}, G_{B} \in \widetilde{\beta}, \exists H_{C} \in \widetilde{\beta} \ni H_{C} \sqsubseteq F_{A} \sqcap G_{B} .
\end{aligned}
$$

Remark $7 \tilde{\beta}$ which is in Proposition 4 is a soft filter base.

Remark 8 It is clear that, every soft filter is a soft filter base.

Example 10 Let $\Phi \neq F_{A} \in \mathcal{S}(X, E)$. Then, the family $\tilde{\beta}=\left\{F_{A}\right\}$ is a soft filter base over $X$.

Example 11 Let $(X, \tilde{\tau})$ be a soft topological space and $e_{F} \stackrel{\sim}{\in} \tilde{X}$. The soft neighbourhood base $\mathcal{G}_{\tilde{\tau}}\left(e_{F}\right)$ is a soft filter base over $X$.

$\left.b_{1}\right)$ Clearly, $\quad \mathcal{G}_{\tilde{\tau}}\left(e_{F}\right) \neq \emptyset$. For every $H_{C} \in \mathcal{G}_{\tilde{\tau}}\left(e_{F}\right)$, $e_{F} \tilde{\in} H_{C}$. Then, $H_{C} \neq \Phi$. Hence, we obtain $\Phi \notin \mathcal{G}_{\tilde{\tau}}\left(e_{F}\right)$. $\left.b_{2}\right)$ Let $G_{B}, H_{C} \in \mathcal{G}_{\tilde{\tau}}\left(e_{F}\right)$. Since $G_{B}, H_{C} \in \mathcal{N}_{\tilde{\tau}}\left(e_{F}\right)$, we get $G_{B} \sqcap H_{C} \in \mathcal{N}_{\tilde{\tau}}\left(e_{F}\right)$. By Definition 13, there exists a $K_{D} \in \mathcal{G}_{\tilde{\tau}}\left(e_{F}\right)$ such that $K_{D} \sqsubseteq G_{B} \sqcap H_{C}$. Hence we get $\mathcal{G}_{\tilde{\tau}}\left(e_{F}\right)$ is a soft filter base of soft neighbourhoods filter $\mathcal{N}_{\tilde{\tau}}\left(e_{F}\right)$ by Definition 16.
Author contribution All authors contributed equally and significantly in writing this paper. All authors read and approved the final manuscript.

Conflict of interest The authors declare that they have no competing interests.

Open Access This article is distributed under the terms of the Creative Commons Attribution License which permits any use, distribution, and reproduction in any medium, provided the original author(s) and the source are credited.

\section{References}

1. Zadeh, L.A.: Fuzzy sets. Inf. Control. 8, 338-353 (1965)

2. Pawlak, Z.: Rough sets. Int. J. Comput. Inf. Sci. 11, 341-356 (1982)

3. Molodtsov, D.: Soft set theory-first results. Comput. Math. Appl. 37, 19-31 (1999)

4. Molodtsov, D.: The theory of soft sets. URSS Publishers, Moscow (in Russian) (2004)

5. Shabir, M., Naz, M.: On soft topological spaces. Comput. Math. Appl. 61, 1786-1799 (2011)

6. Çağman, N., Karataş, S., Enginoğlu, S.: Soft topology. Comput. Math. Appl. 62, 351-358 (2011)

7. Aygünoğlu, A., Aygün, H.: Some notes on soft topological spaces. Neural Comput. Appl. 21(1), 113-119 (2012)

8. Min, W.K.: A note on soft topological spaces. Comput. Math. Appl. 62, 3524-3528 (2011)

9. Zorlutuna, İ., Akdağ, M., Min, W.K., Atmaca, S.: Remarks on soft topological spaces. Ann. Fuzzy Math. Inf. 3(2), 171-185 (2012)

10. Hussain, S., Ahmad, B.: Some properties of soft topological spaces. Comput. Math. Appl. 62, 4058-4067 (2011)

11. Pazar Varol, B., Aygün, H.: On soft hausdorff spaces. Ann. Fuzzy Math. Inf. 5(1), 15-24 (2013)

12. Yüksel, S., Dizman, T., Yildızdan, G., Sert, U.: Application of soft sets to diagnose the prostate cancer risk. J. Inequal. Appl. 229, (2013)

13. Majumdar, P., Samanta, S.K.: Similarity measure of soft set. New Math. Nat. Comput. 4(1), 1-12 (2008)

14. Çă̆man, N., Enginoğlu, S.: Soft set theory and uni-int decision making. Eur. J. Oper. Res. 207, 848-855 (2010) 